\title{
Distribución de la brioflora en el gradiente vertical (suelo-dosel) y la selectividad de habitats en Tarapacá (Amazonas, Colombia).
}

\author{
Cesar Augusto Ruiz-A
}

Programa de Maestría en Biología. Línea Biodiversidad y Conservación. Instituto de Ciencias Naturales-Universidad Nacional de Colombia. Bogotá. cesarruiz1@excite.com

\section{Jaime Aguirre Ceballos}

Instituto de Ciencias Naturales-Universidad Nacional de Colombia. Programa de Doctorado en Biología-Línea de Biodiversidad y Conservación. Apartado aéreo 7495, Bogotá D.C. Colombia.jaguirre@unal.edu.co

Resumen: En los diferentes tipos de paisaje del corregimiento de Tarapacá (Amazonas-Colombia) se estudió la distribución vertical de los briófitos sobre los forofítos, encontrándose que alrededor del $41 \%$ de las especies son exclusivas del dosel y el 35\% exclusivas del sotobosque. Además, se analiza la selectividad de las especies de briófitos por diferentes tipos de hábitats (base de tronco, corteza baja, corteza alta, ramas bajas, ramas altas, epífilos, roca, hojarasca, suelo y troncos caídos en descomposición) y sus formas de crecimiento. En todos los tipos de paisaje, la selectividad de la brioflora fue mayor sobre la corteza baja de los árboles y los hábitats del dosel (hojas y ramas del dosel a más de $18 \mathrm{~m}$ de altura sobre el suelo), con las formas de crecimiento más comunes (felpas lisas, felpas rugosas e hilos) y el mayor número de especies (especialistas y/o generalistas). Los resultados obtenidos para Tarapacá, fueron comparados con otras regiones similares.

Abstract: In the different types of landscape of Tarapacá (Amazonas-Colombia) the vertical
distribution of the bryophytes was studied, being found that around $41 \%$ of the species are exclusive
of the canopy and $35 \%$ exclusive of the understory. Furthermore, there is analyzed the selectivity of
the bryophytes species for different types of habitats (trunk, low bark, high bark, low branches, high
branches, foliicolous, rocks, soil and rotten trunks fallen down) and their growth forms. In all the
landscape types, the selectivity of the bryophytes was greater for the low bark and the habitats of the
canopy (leaves and canopy branches more than $18 \mathrm{~m}$ high), with the more common growth forms
(smooth mats, rough mats and threads) and the larger number of species (,,Specialist“"o,,Generalist“).

The results obtained for Tarapacá were compared with other similar regions. 


\section{INTRODUCCIÓN}

Aspectos de la ecología de los briófitos como su establecimiento en diferentes tipos de bosques, su distribución a lo largo del gradiente altitudinal, su distribución vertical en el forofito, la selectividad de las especies por los tipos de hábitats y la relación de éstas con los tipos de vegetación fanerógamica, han sido poco abordados en Colombia; menos aún en la región amazónica. El tema ha sido tratado por Wolf (1993), Aguirre \& Ruiz (2001), Ruiz (2001) y Ruiz \& Aguirre (2003a, 2003b, 2003c) en Colombia. Richards (1934, 1954, 1984), Florschutz-de Waard \& Bekker (1987), Cornelissen \& Steege (1989), Cornelissen \& Gradstein (1990), Gradstein et al. (1990, 2001) y Gradstein (1992, 1995), en otras regiones tropicales.

La presente contribución se ocupa del análisis de la distribución vertical, desde el suelo hasta el dosel, de las especies de briófitos (musgos y hepáticas) en los diferentes tipos de paisaje en Tarapacá (Amazonas - Colombia). Se analiza la selectividad de la brioflora por los diferentes tipos de hábitats y la relación con sus formas de crecimiento. Se efectúa una comparación de los resultados con contribuciones de regiones similares.

\section{AREA DE ESTUDIO}

Tarapacá (Amazonas-Colombia) con un área de $1100 \mathrm{Km}^{2}$, está situado entre $02^{\circ} 52^{\prime \prime}$ de latitud sur y $69^{\circ} 45^{\prime \prime}$ de longitud oeste, limita al norte con la línea divisoria de aguas entre el río Puré y el río Putumayo, por el oriente con el río Alegría hasta su desembocadura en el río Putumayo, por el occidente con el área forestal del caserío Puerto Palmas y por el sur con el río Putumayo (Cárdenas et al. 1999).

El clima es cálido húmedo (sistema de CaldasLang) con temperaturas homogéneas que oscilan entre 24 y $26^{\circ} \mathrm{C}$. La precipitación es unimodal y alcanza los $3000 \mathrm{~mm} /$ año. El periodo seco ocurre entre los meses de julio y septiembre (Rangel \& Luengas 1997).

\section{UNIDADES DE PAISAJE}

Las unidades de paisaje que conforman las áreas forestales fueron definidas por Cárdenas et al. (1999):

Gran paisaje: „Planicies Disectadas Plio pleistocenicas Amazónicas sedimentarias":

En esta unidad de gran paisaje se agrupan las subunidades de bosque con vegetación natural, no sujeta a inundaciones estacionales, situadas en zonas altas, con superficies fuertemente onduladas y con cimas amplias a subagudas. El relieve es fuertemente ondulado a quebrado, con pendientes entre 20 y $40 \%$. Fuertes procesos de deslizamiento y solifuxión, muy susceptibles a la erosión. Unidad de suelos: Complejo Typic Hapludox y Typic paleudults.

Gran paisaje: „Llanura aluvial del río Putumayo (río Andinense)"

En esta unidad de gran paisaje se agrupan las subunidades de bosque con vegetación natural sujeta a la inundación estacional en las temporadas de aumento del nivel de los ríos. Presenta las subunidades:

- Plano de inundación actual. Plano inundable frecuente y ocasional. Nivel freático alto y fluctuante; hidromorfismo temporal. Superficies de acumulación de sedimentos. Unidad de suelos: Asociaciones Fluventic Eutropepts y Tropic Fluvaquents.

- Plano de inundación subactual. Relieve Plano - cóncavo, estrecho, inundable con frecuencia pero de corta duración. Hidromorfismo. Unidad de suelos: Complejo Aeric Tropic Fluvaquents, Histic Tropaquepts y Fluventic Dystroprepts.

- Valles aluvio - coluviales. Planos inundables frecuentemente, con acumulación actual de sedimentos aluviales y coluviales. Superficies estrechas y alargadas.

Unidad de paisaje Intervenida „Pista de Aterrizaje“. Ubicada cerca de la cabecera de Tarapacá. Son áreas intervenidas con vegetación secundaria, por intervención 
antrópica. Son relictos de bosque correspondientes al paisaje „Planicies Disectadas Plio - pleistocenicas Amazónicas sedimentarias", actualmente han sido sometidas a fragmentación y tala intensiva para construir viviendas y una pista de aterrizaje. Sus características fisiográficas son similares a las de las Planicies Disectadas Plio-pleistocenicas Amazónicas Sedimentarias, y la diferencia radica en la presencia de una cobertura vegetal transformada y abierta.

\section{MÉTODOS}

La fase de campo se realizó del 20 de febrero al 26 de marzo de 1999. Se efectuaron colecciones de musgos, hepáticas y líquenes. Se siguieron los lineamientos de Cornelissen \& Steege (1989) y Wolf (1993), realizando la colecta en 56 levantamientos (24 levantamientos en las unidades fisiográficas del gran tipo de paisaje „Planicies Disectadas Plio-Pleistocenicas Amazónicas sedimentarias“, 20 en las unidades fisiográficas de la „Llanura aluvial del río Putumayo“ y 12 en una unidad altamente intervenida „PISTA“, aledaña al casco urbano de Tarapacá) seleccionados para el estudio de la vegetación superior y delimitados según la fisonomía de cada una de las regiones (Ruiz 2001).

Los briófitos y líquenes se colectaron bajo la numeración del primer autor (CAR No 10-1118). Se depositaron en el Herbario Nacional Colombiano (COL) con duplicados en el Herbario Amazónico Colombiano (COAH). En el censo (presencia y cobertura) se tomó información sobre el sustrato (epífitas, epilíticas, terrestres), hábito (epífitas, epífilas, terrícolas, saxícolas), forma de crecimiento y abundancia según la escala de cobertura- abundancia de Braun-Blanquet (1979).

Para el análisis de la distribución vertical (desde el suelo hasta el dosel) se tomaron los resultados (presencia y cobertura) de los muestreos realizados en 280 árboles censados.

La presencia y cobertura de las especies en los hábitats (para definir las especies especialistas o generalistas), se estudio de acuerdo con Cornelissen \& Steege (1989) quienes definen los hábitats como sigue: Base de tronco $(\mathrm{Bt})$, corteza baja (Cort, hasta $2 \mathrm{~m}$ de altura), ramas del sotobosque (Ram, hasta 9-10 m de altura), rocas (Roc), suelo (Suel), hojarasca (Hoj), troncos caídos descompuestos (Tdc), corteza alta (Cortal, de $4 \mathrm{~m}-18 \mathrm{~m}$ ), epífilos del dosel (Epif, hojas del dosel $>20 \mathrm{~m}$ ), ramas altas (Ramal, a más de 20 metros de altura).

En toda el área y en cada tipo de paisaje se calcularon las frecuencias relativas (FR), para determinar la selectividad de las especies por un tipo de hábitat. Además, se aplicó el análisis de ordenación de hábitats DCA (Análisis de Correspondencia „Detrended Correspondence Análisis“). El arreglo se basó en los promedios de cobertura en el „Espacio de ordenación“.

El estudio de las formas de crecimiento se realizó de acuerdo con los planteamientos de Mägdefrau (1982), Studlar (1982), Richards (1984), Cornelissen \& Steege (1989) y Churchill \& Linares (1995). En la zona se tomaron en cuenta: Cespitosas bajas $(\mathrm{Cc})$, cespitosas altas $(\mathrm{Ca})$, cojines (Coj), hilos o fibras (Hi), felpas lisas (Fl), felpas rugosas $(\mathrm{Fr})$, horizontales $(\mathrm{H})$, taloides $(\mathrm{T})$, péndulas $(\mathrm{P})$, dendroides no dorsiventrales $(\mathrm{A})$ $\mathrm{y}$ formas delgadas (B).

Los resultados obtenidos se compararon con las contribuciones de Gradstein et al (2001) para Monteverde-Costa Rica y Cornelissen \& Steege (1989) para Mabura Hill - Guayana.

\section{RESULTADOS Y DISCUSIÓN}

\section{FORMAS DE CRECIMIENTO}

GLOBALES: Más del 50\% de las especies de briófitos de Tarapacá exhiben formas de crecimiento: felpas lisas (Fl, 29 especies) e hilos (Hi, 22). Le siguen: cespitosas cortas (Cc, 16), felpas rugosas $(\mathrm{Fr}, 12)$ y formas horizontales $(\mathrm{H}$, 10) (Figura 1).

Los cojines (Coj, 3), cespitosas altas $(\mathrm{Ca}, 2) \mathrm{y}$ formas péndulas $(\mathrm{P}, 2)$, son las menos frecuentes. 


\section{POR TIPOS DE PAISAJE:}

A. „Planicies Disectadas PliopleistocenicasAmazónicas Sedimentarias“. En la figura 2, se observa la distribución de las especies según las diferentes formas de crecimiento. La mayoría presentan las formas felpas lisas (Fl-39 especies) e hilos (Hi-32). Otras formas importantes son: dendroides no dorsiventrales (A-20), cespitosas cortas (Cc-18), felpas rugosas (Fr-17) y formas horizontales (H-16). Talosas (T-2), formas péndulas (P-0) y cespitosas altas $(\mathrm{Ca}-0)$ son las menos frecuentes en esta unidad de paisaje.

B. „Llanuras Aluviales del Rió Putumayo“. La figura 3 muestra, las diferentes formas de crecimiento de las especies. La mayoría presentan formas felpas lisas (Fl-32 especies), dendroides no dorsiventrales (A-21) e hilos (Hi19). Les siguen en orden de importancia las cespitosas cortas (Cc-19), horizontales (H-12) y formas delgadas (B-10). Son menos frecuentes en esta unidad de paisaje, cojines (Co-5), formas péndulas (P-3), felpas rugosas (Fr-3) y cespitosas altas $(\mathrm{Ca}-0)$.

C. „Unidad con alta intervención antrópica“ (PISTA). En la figura 4, se observa lo que ocurre en este paisaje. La forma de crecimiento que agrupa el mayor número de especies es felpa lisa (Fl-17 especies). Siguen en orden de importancia: Las formas Horizontales (H-5) y cespitosas cortas (Cc-5). Cojines (Co) y cespitosas altas (Ca) están bien representadas en este paisaje.

D. Para la brioflora de Tarapacá se reportan 11 formas de crecimiento o desarrollo. Algunas especies pueden presentar según las condiciones ambientales, cierta plasticidad adoptando más de una forma de crecimiento (During 1979, Birse 1957).

E. Se observa en las figuras 2 y 3 que, a excepción del paisaje ,Unidad con alta intervención antrópica" (PISTA), no hay diferencias significativas entre la distribución de las formas de vida en los tipos de paisaje. Las formas postradas son dominantes (en número de especies) en los paisajes Planicies Disectadas Pliopleistocenicas-Amazónicas Sedimen- tarias“ y "Llanuras Aluviales del Rió Putumayo".

F. La figura 4 muestra que en el paisaje „Unidad con alta intervención antrópica“ (PISTA) son importantes las formas cespitosas altas (Ca) y los cojines (Co). Esta situación se produce por el efecto de la intervención por tala. En los demás paisajes, las especies cespitosas altas y cojines son escasas, situación similar a la que ocurre en otros bosques tropicales de tierras bajas descritos por Cornelissen \& Steege (1989).

G. Las formas de crecimiento de los briófitos en bosques de Tarapacá responden a las condiciones ambientales (Ruiz, 2001) y a un patrón de dominancia común a los bosques de tierras bajas (Figura 5), donde la felpa lisa (Fl) e hilos (Hi) son las más importantes y comunes entre las especies (Cornelissen \& Steege 1989, Cornelissen \& Gradstein 1990 y Gradstein et al. 2001). De acuerdo con During (1979), Mägdefrau (1982), Studlar (1982), Richards (1984), Cornelissen \& Steege (1989) y Ruiz \& Aguirre (2003a), las formas de crecimiento de los briófitos están estrechamente relacionadas con sus estrategias de vida y sus necesidades o exigencias de luz y humedad.

During (1979) destaca que las formas de crecimiento es tan solo uno de los tantos aspectos que se toman en cuenta para determinar las estrategias de vida de las especies de briófitos; otros incluyen el conocimiento de su ciclo de vida, su tipo de reproducción dominante (sexual o asexual), el conocimiento de sus estrategias de dispersión y su ecofisiología. Muchos de estos aspectos son desconocidos para la mayoría de las especies, y los trabajos en la biología de los briófitos son muy puntuales y escasos.

\section{DISTRIBUCIÓN VERTICAL}

RESULTADOS GLOBALES: El 65\% de las especies (Figura 6) se establecen en el dosel o en zonas intermedias del dosel y el sotobosque. E1 35\% en los sectores bajos de los árboles (base de tronco, cortezas bajas y medias) y ocasionalmente en porciones altas de los mismos (corteza alta y ramas bajas del dosel). 
De las especies con formas de crecimiento dominantes felpa lisa e hilos (Figura 6), el 80\% se establecen de manera exclusiva en el dosel y un $20 \%$ en porciones altas, medias y bajas del tronco del árbol.

Las especies con estas formas de crecimiento responden a las condiciones de alta iluminación y baja humedad que se presenta en el dosel de bosque, criterio ya expuesto por Birse (1957), Barkman (1958) y Gradstein (1995). Además, con estos resultados, se corrobora la afirmación de Gradstein \& Cornelissen (1989) y Gradstein (1995), en cuanto a que algo más del 50\% de la diversidad de briófitos de un bosque tropical se encuentra confinada en el dosel.

El 100\% de las especies cespitosas altas (Ca) y cojines (Co), son exclusivas de la base de tronco, corteza baja y media (Figura 6). Respondiendo así a las condiciones de alta humedad y baja intensidad de luz que predomina en dichas zonas.

Las especies con formas: felpas rugosas (Fr), horizontales $(\mathrm{H})$, dendroides no dorsiventrales (A) y formas delgadas (B), de escasa representación, se distribuyen de manera uniforme en diferentes sectores del árbol, con especial selectividad hacia las partes medias $\mathrm{y}$ bajas del dosel.

La forma péndula (P) es exclusiva de las partes bajas del dosel (primeras ramas de los árboles).

Las epífilas en un $45 \%$ se presentan de manera exclusiva en las partes medias y altas del dosel. El resto (55\%) se distribuye desde el sotobosque (3 metros de altura) hasta las porciones inferiores del dosel (hasta 19 metros), estableciéndose según sus necesidades de humedad e intensidad de luz (Gradstein et al. 1990).

RESULTADOS POR TIPO DE PAISAJE:

A. En el paisaje"Planicies Disectadas Pliople is tocen ic a s - A m azón ic as Sedimentarias" (Figura 7) el 55\% de las especies de briófitos se establecen en el dosel y el $45 \%$ en porciones bajas de los árboles.

El 77\% de las especies con formas felpa lisa (Fl) e hilos (Hi) se establecen de manera exclusiva en el dosel. El 14\% en porciones medias y bajas de los troncos de los árboles, y un $9 \%$ ocurre de manera exclusiva en base de tronco y corteza baja (menos de $4 \mathrm{~m}$ de altura).

El 70\% de las especies con formas de crecimiento cespitosas cortas, felpas rugosas, formas delgadas, cojines y talosas, son exclusivas de corteza baja, base de tronco y suelo.

B. La figura 8, muestra la distribución vertical de las especies para el tipo de paisaje „Llanuras Aluviales del Rió Putumayo". El 58\% de las especies se establecen en el dosel y el $42 \%$ se encuentran en porciones medias y bajas de los árboles.

El 50\% de las especies con formas de crecimiento dominantes felpas lisas (F1), dendroides no dorsiventrales (A) e hilos (Hi), se hallaron de manera exclusiva en el dosel. El $15 \%$ en porciones medias de los troncos de los árboles, y un $35 \%$ se establecen de manera exclusiva en ramas bajas del sotobosque y corteza baja.

Cespitosas cortas (Cc-10 especies), formas delgadas (B-8), formas talosas (T-6), cojines (Co$5)$, formas péndulas (P-3) y felpas rugosas (Fr3 ), son comunes en zonas bajas de los árboles y del bosque.

C. La figura 9, presenta la distribución vertical de las especies en el tipo de paisaje „Unidad con alta intervención antrópica“" (PISTA). Menos del 10\% de las especies se establecen en el dosel. El 90\% restante, aparecen en porciones medias y bajas de los árboles y en hojas y ramas de arbustos e hierbas.

En este paisaje las hepáticas epífilas reducen su diversidad en un $90 \%$, solo se registran 6 especies. Debido a la tala constante que ha sufrido este bosque, la riqueza en el dosel y la diversidad de microhábitats disminuyen drásticamente. 
La mayor diversidad de briófitos se encuentra en las zonas bajas de los árboles, donde predominan las formas de crecimiento cespitosas y semierectas, respondiendo a las nuevas condiciones de humedad e intensidad lumínica.

Las formas de crecimiento dominantes felpas lisas y horizontales, se hallaron de manera esporádica en el dosel con el $8 \%$ de sus especies. El 92\% se establecen en porciones bajas de los árboles.

D. Los paisajes analizados, con excepción de la „Unidad con alta intervención antrópica“" (PISTA), presentan el mismo patrón de distribución vertical; donde aproximadamente el $40 \%$ de las especies se establecen exclusivamente en el dosel (Figura 10).

E. Si se comparan los resultados de diversidad vertical obtenidos (Figura 10) con regiones como Monteverde - Costa Rica (Gradstein et al. 2001) y Mabura Hill - Guayana (Cornelissen \& Steege, 1989), se observan similitudes. Por ejemplo, el mismo patrón de diversidad vertical que se ha expuesto anteriormente. Algo más del 50\% de la diversidad de briófitos, de los bosques comparados, se encuentra en el dosel o en zonas inmediatamente inferiores.

\section{SELECTIVIDAD DE HABITATS}

A. En la figura 11 se exponen los resultados de selectividad de hábitats para toda el área de estudio (a excepción del paisaje ,Unidad con alta intervención antrópica"). El hábitat que presenta la mayor cantidad de especies (totales y exclusivas) es corteza baja (Cort- 57 especies totales- 36 especies exclusivas). Le sigue en orden de importancia hojas del dosel (Epifil-2614), ramas altas (Ramal-28-1), corteza alta (Cortal-18-1), troncos caídos descompuestos (Tdc-13-1), ramas bajas del sotobosque (Ram20-1), base de tronco (Bt-2-0) y Suelo (Suel-2$0)$.

B. En el paisaje „Unidad con alta intervención antrópica“" (Figura 12), la mayor cantidad de especies se establece en el hábitat corteza baja
(Cort-16 especies totales- 12 especies exclusivas). Le sigue en orden de importancia hojas de arbustos (Epifil-4-4), suelo (Suel-4-3), troncos caídos descompuestos (Tdc-4-1), ramas bajas del sotobosque (Ram-2-2) y rocas (Roc-10 ). Además, estos hábitats presentan la mayor diversidad en formas de crecimiento.

C. La mayor cantidad de especies exclusivas se encuentran en corteza baja, en segundo lugar se encuentran hojas del dosel y ramas del dosel (Anexo 1)

D. Son „especialistas“ de hábitats del dosel: Microlejeunea bullata, Microlejeunea epiphylla, Leptolejeunea elliptica, Leptolejeunea tridentata, Radula cf. angulata, Odontolejeunea rhomalea, Crossomitrium acuminatum y Lepidolejeunea grossepapulosa, en hojas del dosel. Lejeunea aff. flava, Archilejeunea crispistipula, Calypogeia densifolia y Cyclolejeunea peruviana, en corteza alta. Zelometeorium patulum, Anoplolejeunea conferta y Isopterygium tenereum, en ramas altas. Las formas de crecimiento que exhiben estas especies (hilos y felpas lisas) responden a condiciones de alta luminosidad y baja humedad, que se dan en el dosel de los bosques.

Cornelissen \& Steege (1989), Gradstein et al.(1990), Cornelissen \& Gradstein (1990), Gradstein (1995) y Gadstein et al. (2001) afirman que la mayor diversidad en formas de crecimiento y número de especies se presenta en el dosel, principalmente en ramas altas. Los presentes resultados concuerdan con los expuestos en estas contribuciones (Figura 13), aunque se evidencian diferencias; que demuestran que el muestreo fue más intensivo en las secciones del sotobosque.

E. Son „especialistas“ de hábitats del sotobosque, los taxa: Arachniopsis diacantha, Calymperes afzelli, Calymperes lonchophyllum, Cyrtohypnum frontinoae, Chryso-hypnum diminutivum, Ectropothecium leptochaeton, Lepidopilum frondosum, Lepidopilum surinamense, Leucobryum martianum, Micropterygium leophyllum, Micropterygium pterygophyllum, Octoblepharum albidum, Octoblepharum pulvinatum, Riccardia aff. 
amazonica, Stictolejeunea squamata, Syrrhopodon hornschuchi, Syrrhopodon incompletus, Syrrhopodon leprieuri, Syrrhopodon ligulatus, Syrrhopodon rigidus y Syrrhopodon simmondsii.

En la zona de estudio el número de especies en los hábitats del sotobosque, es muy alto; pero no superior al que se da en los hábitats del dosel. Las formas de crecimiento semierectas y erectas son de distribución restringida y responden a las condiciones ambientales propias del sotobosque.

F. En el paisaje ,Unidad con alta intervención antrópica" el patrón de selectividad de hábitats es claramente distinto, por su transformación debido a la tala intensiva. En este paisaje los hábitats del dosel están poco representados, y se observa una ausencia total de especies exclusivas en los mismos. El hábitat mejor representado es corteza baja, con especies que exhiben formas de crecimiento erectas (cespitosas altas y cojines). Este paisaje se caracteriza por la dominancia de especies, generalistas“, en cuanto a su preferencia sobre los hábitats.

\section{CONSIDERACIONES FINALES}

1. Para la brioflora de Tarapacá, se reportan 11 formas de crecimiento o desarrollo.

2. Con excepción del paisaje „Unidad con alta intervención antrópica", del 45\% al $55 \%$ del total de las especies de briófitos aparecen de manera exclusiva o semiexclusiva en el dosel de los bosques (Anexo 1). No se presentan diferencias significativas entre los paisajes. Las formas de crecimiento que predominan (en número de especies) son las postradas.

3. Las formas de crecimiento de los briófitos de los bosques de Tarapacá responden a las condiciones ambientales y se ajustan a un patrón de dominancia que es común en los bosques de tierras bajas; la felpa lisa e hilos son las más comunes e importantes, entre las especies que ocurren en el dosel.

4. La menor diversidad se presenta en el sotobosque, dominan las especies con formas de crecimiento semierectas (felpas rugosas y cespitosas cortas), que exigen una mayor humedad y que son tolerantes a variados niveles de luz solar.

5. Cuando se comparan los resultados de diversidad vertical con regiones como Monteverde - Costa Rica (Gradstein et al. 2001) y Mabura Hill-Guyana (Cornelissen \& Steege 1989), hay similaridad, se presenta el mismo patrón, donde algo más del $50 \%$ de la diversidad de briófitos de los bosques comparados se encuentra en el dosel o zonas inferiores.

6. Se presenta un patrón similar en cuanto a la selectividad de hábitats, en todos los tipos de paisaje (a excepción de „Unidad con alta intervención antrópica"). No existen diferencias significativas en la distribución de las especies entre hábitats y formas de crecimiento.

7. La corteza baja es un hábitat que exhibe una alta diversidad de especies y formas de crecimiento; la riqueza de especies en los hábitats del dosel (corteza alta, ramas altas y hojas del dosel) y porciones cercanas, suma algo más del $50 \%$. La mayor cantidad de especies exclusivas siempre se encuentra en corteza baja; en segundo lugar están hojas y ramas del dosel.

8. En el paisaje ,Unidad con alta intervención antrópica" se destaca la desaparición del $80 \%$ de las especies del dosel, del $80 \%$ de las Lejeuneaceae y una reducción en más del $60 \%$ de las especies exclusivas del sotobosque. 
9. Por los resultados expuestos, se puede afirmar que la intervención (por la tala intensiva) de los bosques tropicales afecta la presencia y la selectividad por hábitat de las especies de briófitos, debido a los nuevos microambientes que se generan. Luego de un impacto, como el que se causa por este fenómeno, a la vegetación de fanerógamas las especies de briófitos que permanecen adoptan formas de crecimiento alternativas, como respuesta a las nuevas condiciones generadas.

Agradecimientos: Al Instituto Amazónico de Investigaciones Científicas- SINCHI en especial a Dayron Cárdenas, al Herbario Amazónico Colombiano (COAH). A la Universidad Nacional de Colombia, al Instituto de Ciencias Naturales y al Herbario Nacional Colombiano (COL), por el apoyo prestado al desarrollo de la investigación

\section{Referenciass}

Aguirre, J. \& C. A, Ruiz. 2001. Composición florística de la brioflora de la Serranía del Perijá (Cesar - Colombia): Distribución y ecología. Caldasia 23 (1): 181-201.

Barkman, J. 1958. Phytosociology and ecology of cryptogamic epiphytes. Van Gorcum. Assen. $628 \mathrm{pp}$.

Birse, E.M. 1957. Ecologycal studies on growth-form in bryophytes. II Experimental studies on growth-form in mosses. Journal of Ecology 45: 721-733.

Braun-Blanquet, J. 1979. Fitosociología: bases para el estudio de las comunidades vegetales. Ed. Blume, Madrid. 819p.

Cárdenas, D, C. Marín \& R. López. 1999. Primer informe de avance del Proyecto „Experiencia piloto de Zonificación como Instrumento para la Planificación de las Áreas Forestales en el Corregimiento de Tarapacá (Amazonas)“". Instituto Amazónico de Investigaciones Científicas SINCHI. Santafé de Bogotá. (Inédito).

Churchill, S.P \& E.L. Linares. 1995. Prodomus Bryologiae Novo- Granatensis.introducción a la flora de musgos de Colombia.
Biblioteca José Jerónimo Triana. Tomos I y II. Instituto de Ciencias Naturales. Facultad de Ciencias. Universidad Nacional de Colombia. Bogotá.

Cornelissen, J.H.C \& H.T, Steege. 1989. Distribution and ecology of epiphytic bryophytes and lichens in dry evergreen forest of Guyana. Journal of Tropical Ecology 5:131-150.

Cornelissen, J.H.C \& S.R. Gradstein 1990. On the occurrence of bryophytes and macrolichenes in different lowland rain forest types at Mabura Hill, Guyana. Tropical Bryology 3: 29-35.

During, J.H. 1979. Life strategies of bryophytes: a preliminary review. Lindbergia 5: 2-18.

Florschürtz D,W. \& J.M. Bekker, 1987. A comparative study of the bryophyte flora of different forest types in west Suriname. Cryptogamie, Bryologie et Lichenologie 8: 31-45.

Gradstein, S.R. 1992. Threatened bryophytes of the neotropical rain forest: a status report. Tropical Bryology $6: 83-93$.

Gradstein, S.R. 1995. Bryophyte diversity of the tropical rainforest. Archs. Sci. Geneve 48(1):91-95.

Gradstein, S.R., D. Montfoort \& J.H.C. Cornelissen. 1990. Species richness and phytogeography of the bryophyte flora of the Guianas, with special reference to the lowland forest. Tropical Bryology 2:117126.

Gradstein, S.R., D. Griffin Iii, M.I. Morales \& N.M. Nadkarni. 2001. Diversity and habitat differentiation of mosses and liverworts in the cloud forest of Monteverde, Costa Rica. Caldasia 23 (1): 203-212.

Mägdefrau, K. 1982. Life - forms of bryophytes. Pp. 45-58 in: SMITH, A.J.E. (ed).Bryophyte ecology. Chapman and Hall.

Rangel, E \& B. Luengas. 1997. Clima y aguas del eje Apaporis - Tabatinga. Pag: 49 - 68. en: IGAC - SINCHI - Universidad Nacional de Colombia. Zonificación ambiental para el plan modelo colombo - brasilero (eje Apaporis - Tabatinga). Santafé de Bogotá.

Richards, P.W. 1934. Musci collected by the Oxford expedition to British Guiana in 1929. Bulletin of Miscellaneous information, Kew 1934: 317-337.

Richards, P.W.. 1954. Notes on the bryophyte communities of lowland tropical rain forest, with special reference to Moraballi creek, British Guyana. Vegetatio 5-6:319-328. 
Richards, P.W.. 1984. The ecology of tropical forest bryophytes. En: SCHUSTER, R.M. (ed). New manual of bryology 2: 1233-1270.

Ruiz, C.A.. 2001. Análisis Florístico y de Vegetación de la brioflora del Corregimiento de Tarapacá (Amazonas-Colombia). Tesis de Grado. Departamento de Biología. Universidad Nacional de Colombia. Bogota.

Ruiz, C.A. \& J. Aguirre 2003a. Las comunidades de briófitos y su relación con la estructura de la vegetación fanerogamica, en el gradiente altitudinal de La Serranía Del Perijá (Cesar - Colombia). Tropical Bryology 24: 101-113.

Ruiz, C.A. \& J. Aguirre. (En Prep.) 2003b. Análisis florístico de la brioflora del corregimiento de Tarapacá (Amazonas- Colombia).

Ruiz, C.A. \& J. Aguirre. (En Prensa.) 2003c. Las Comunidades De Briófitos Y Su Relación Con La Vegetación Fanerogamica (Tipos De
Paisaje), En Tarapacá (AmazonasColombia). Caldasia.

Studlar, S.M. 1982. Succession of epiphytic bryophytes near Mountain Lake, Virginia. Bryologist 85: 51-63.

Wolf, J.H.D. 1993. Diversity pattners and biomass of epifhytic bryophyte and lichens along an altitudinal gradient in the northern Andes. Ann. Missouri Bot. Gard. 80: 928-960.

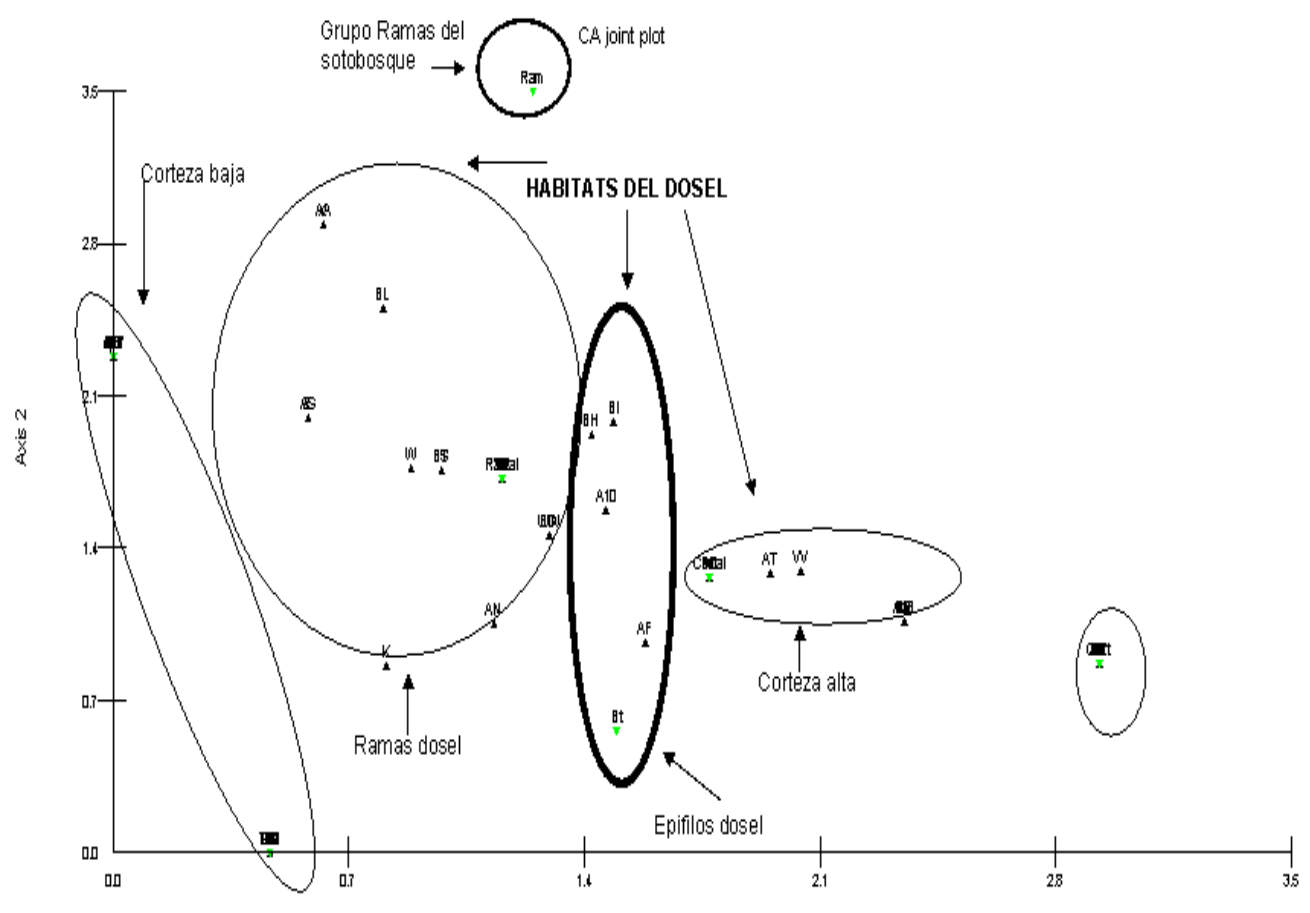

ANEXO 2: Análisis de correspondencia. Selectividad de especies por diferentes tipos de hábitats, en las áreas forestales de Tarapacá Amazonas. Tendencias de especies.

La grafica muestra las tendencias de las especies a agruparse con la mayor frecuencia y el mayor porcentaje de cobertura, sobre un hábitat particular. Se destacan preferencias marcadas (especies ,especialistas“")

Se observa que los grupos con el mayor número de especies „especialistas de hábitat“ corresponden a elementos del dosel (Ramas altas del dosel, Epífilos del dosel y corteza alta). También está bien representado el hábitat Corteza baja con un alto número de „especialistas“. 

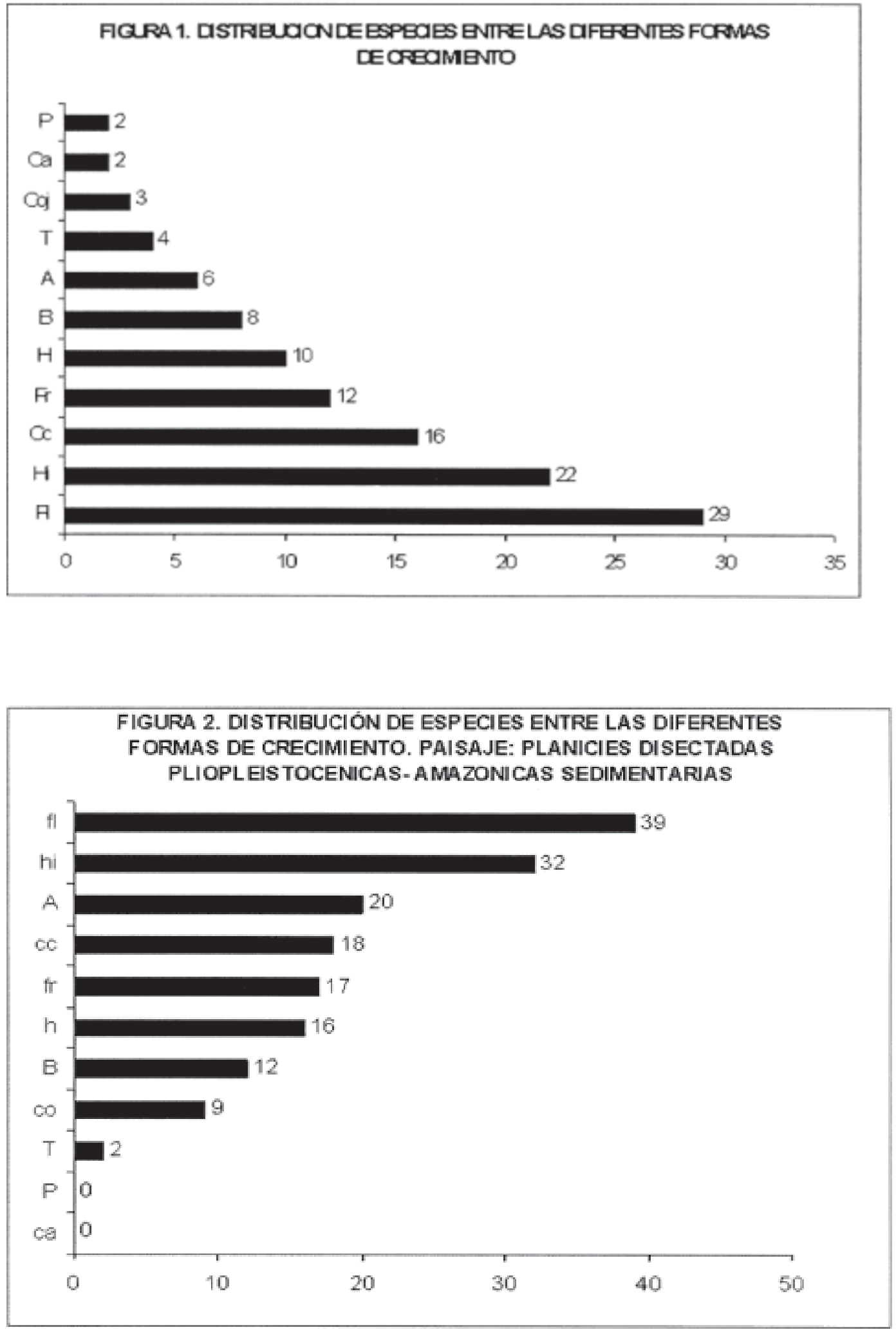

TROPICAL BRYOLOGY 25 (2004) 

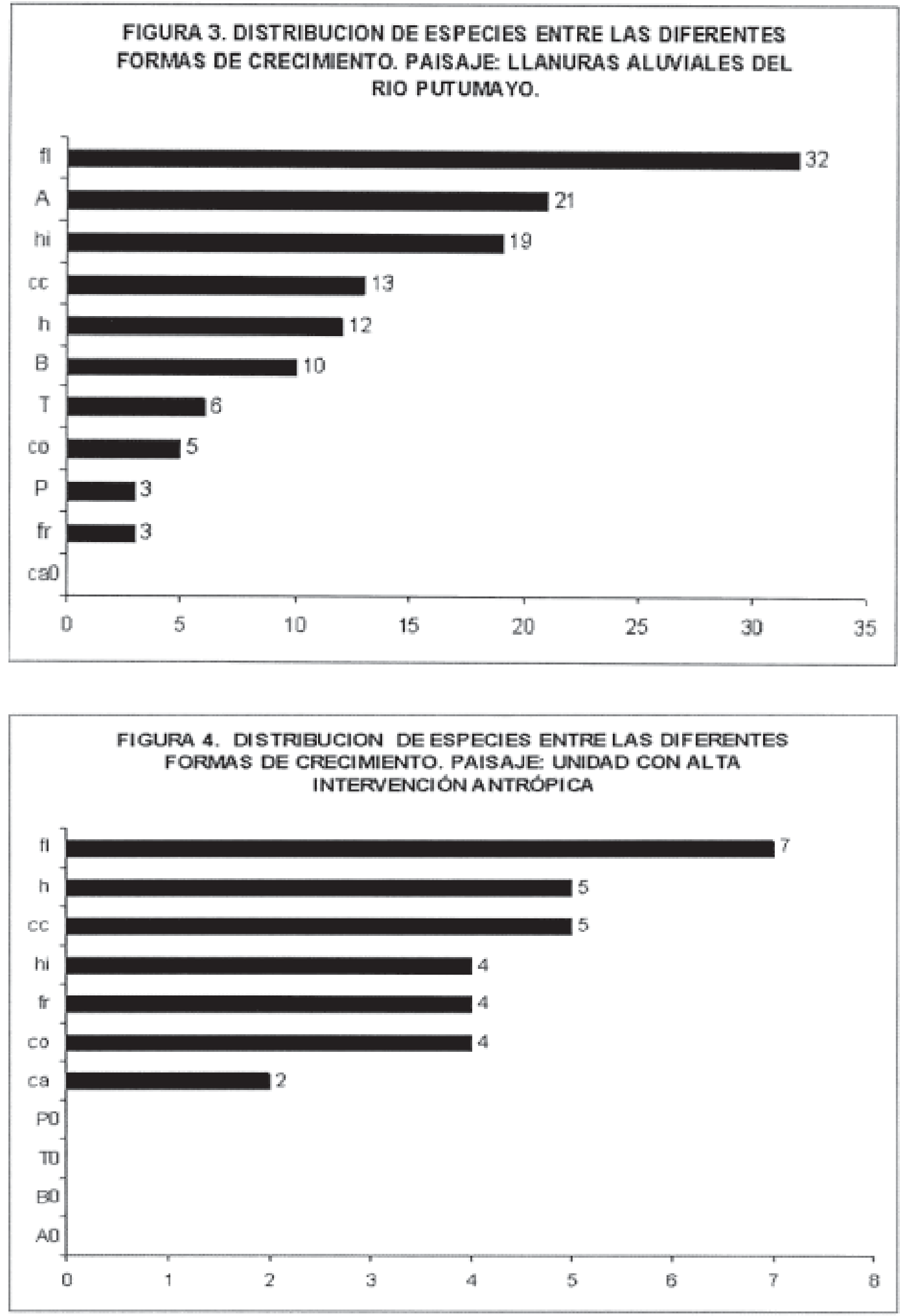

TROPICAL BRYOLOGY 25 (2004) 

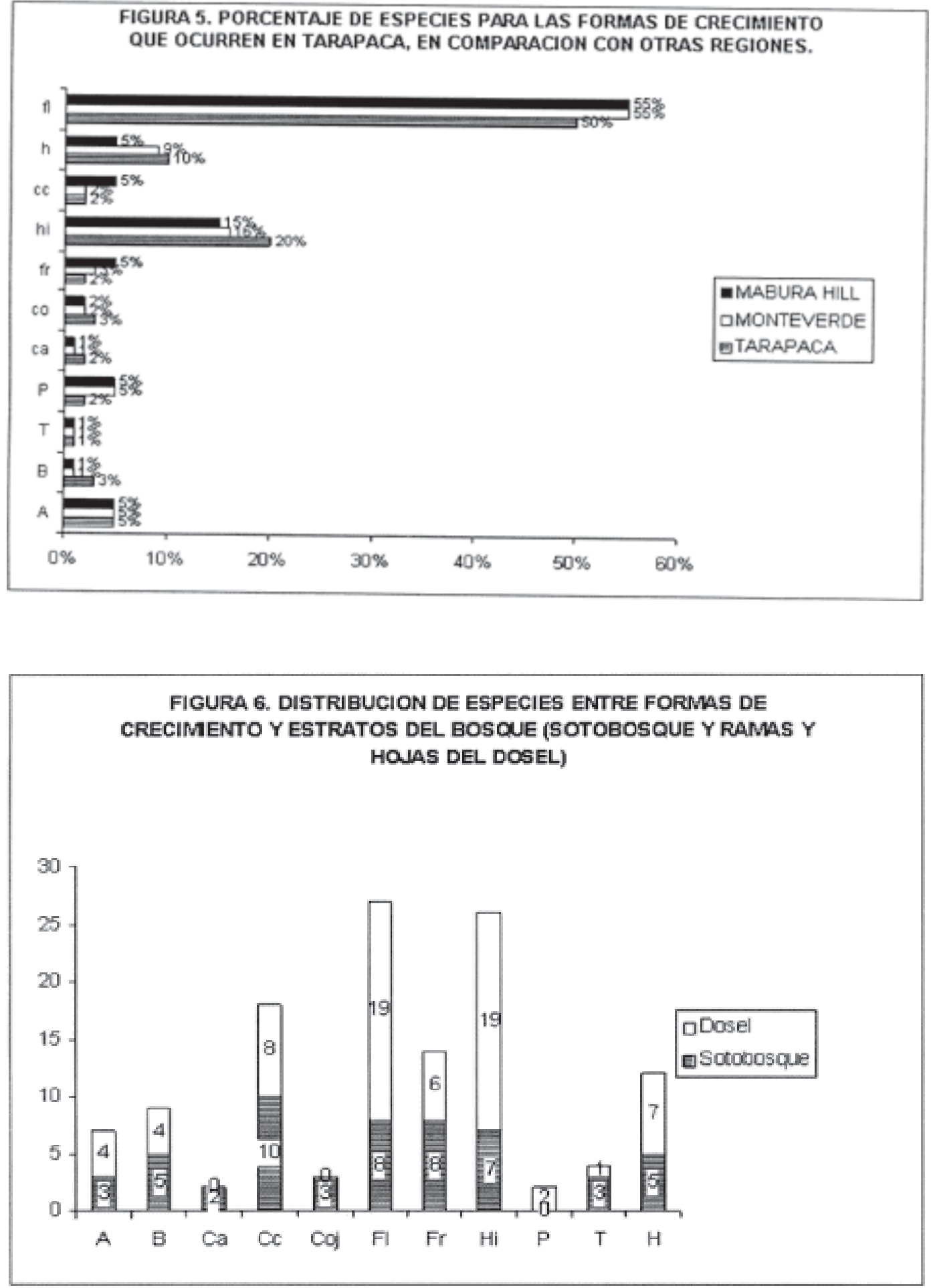

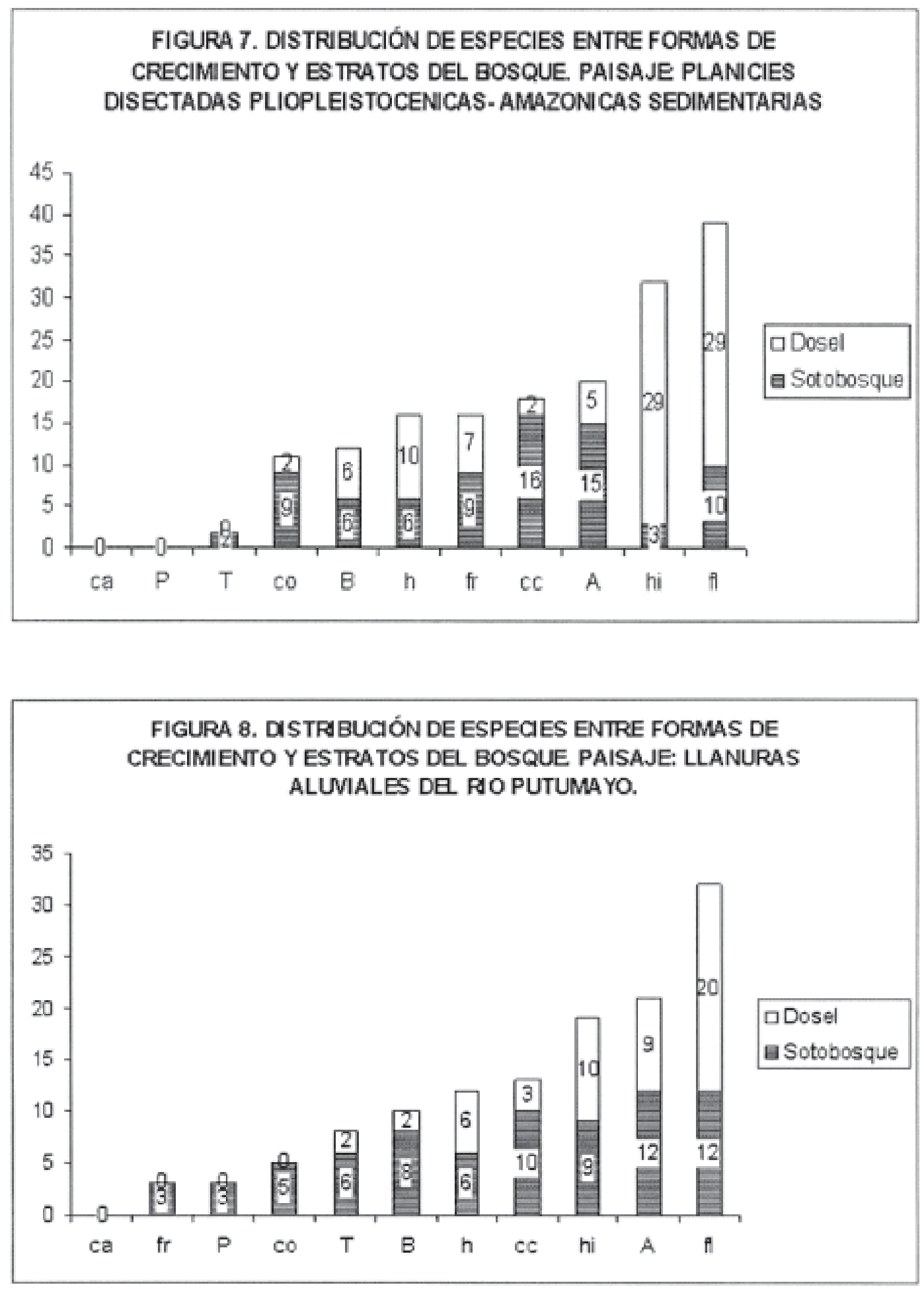

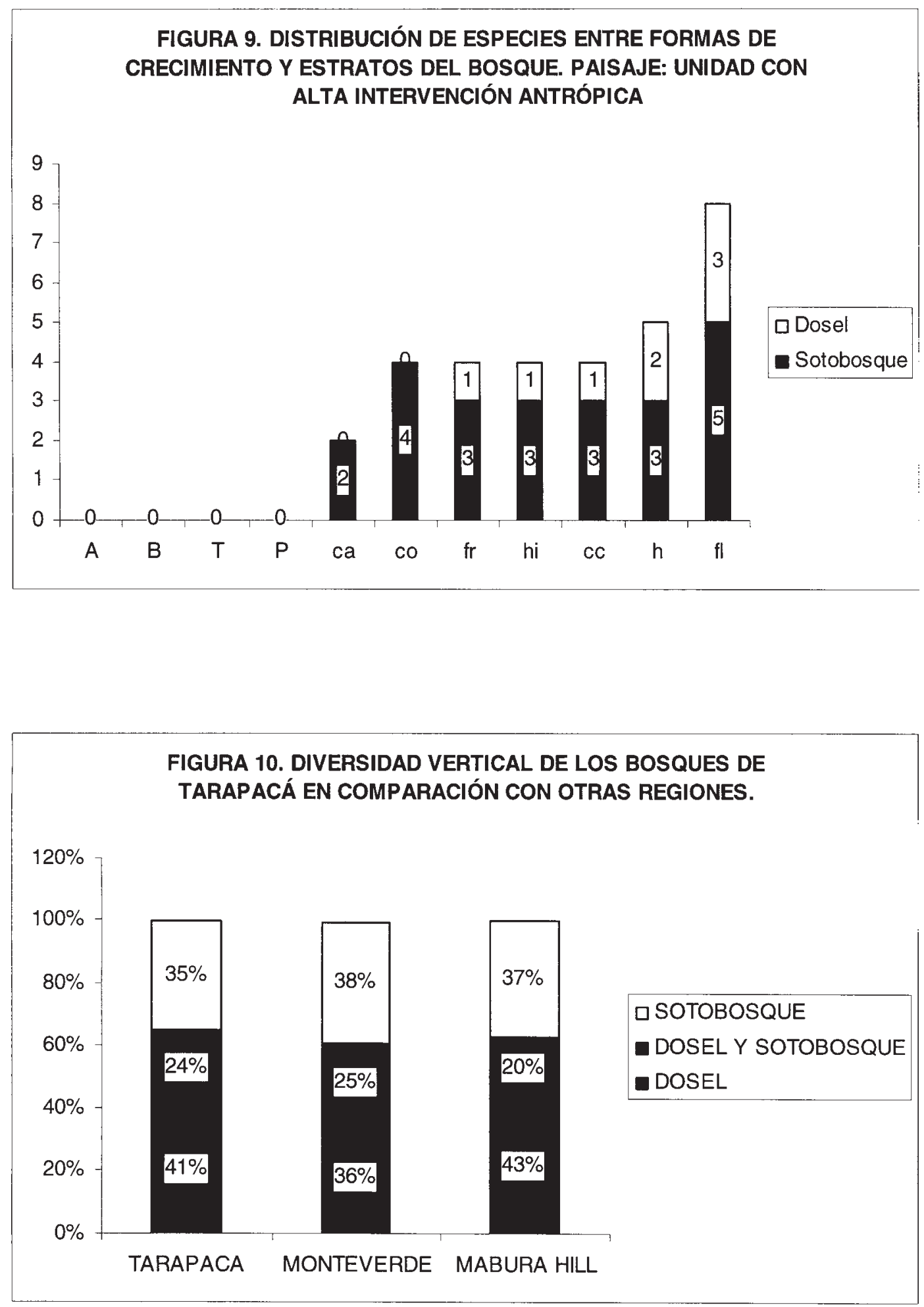

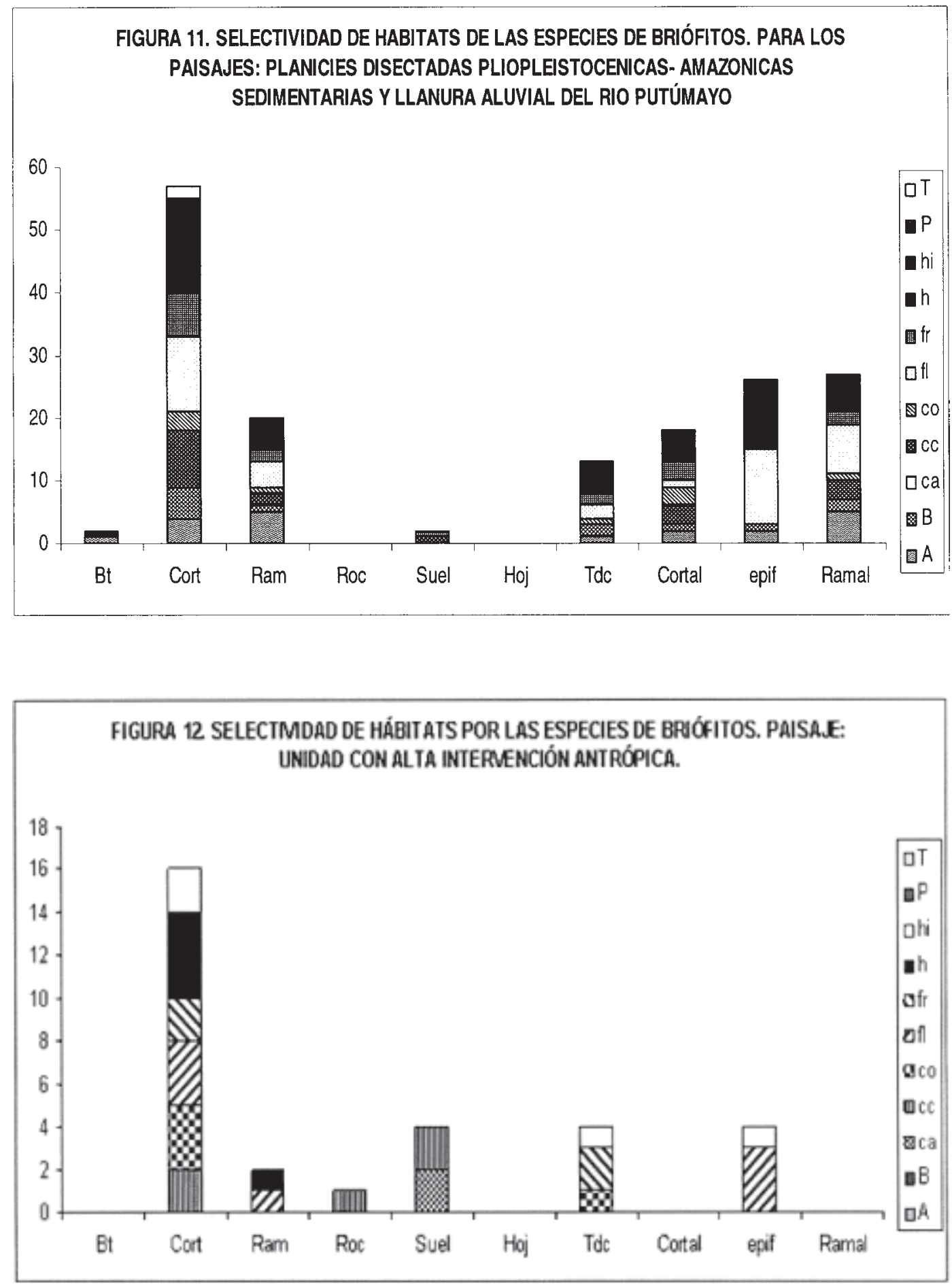


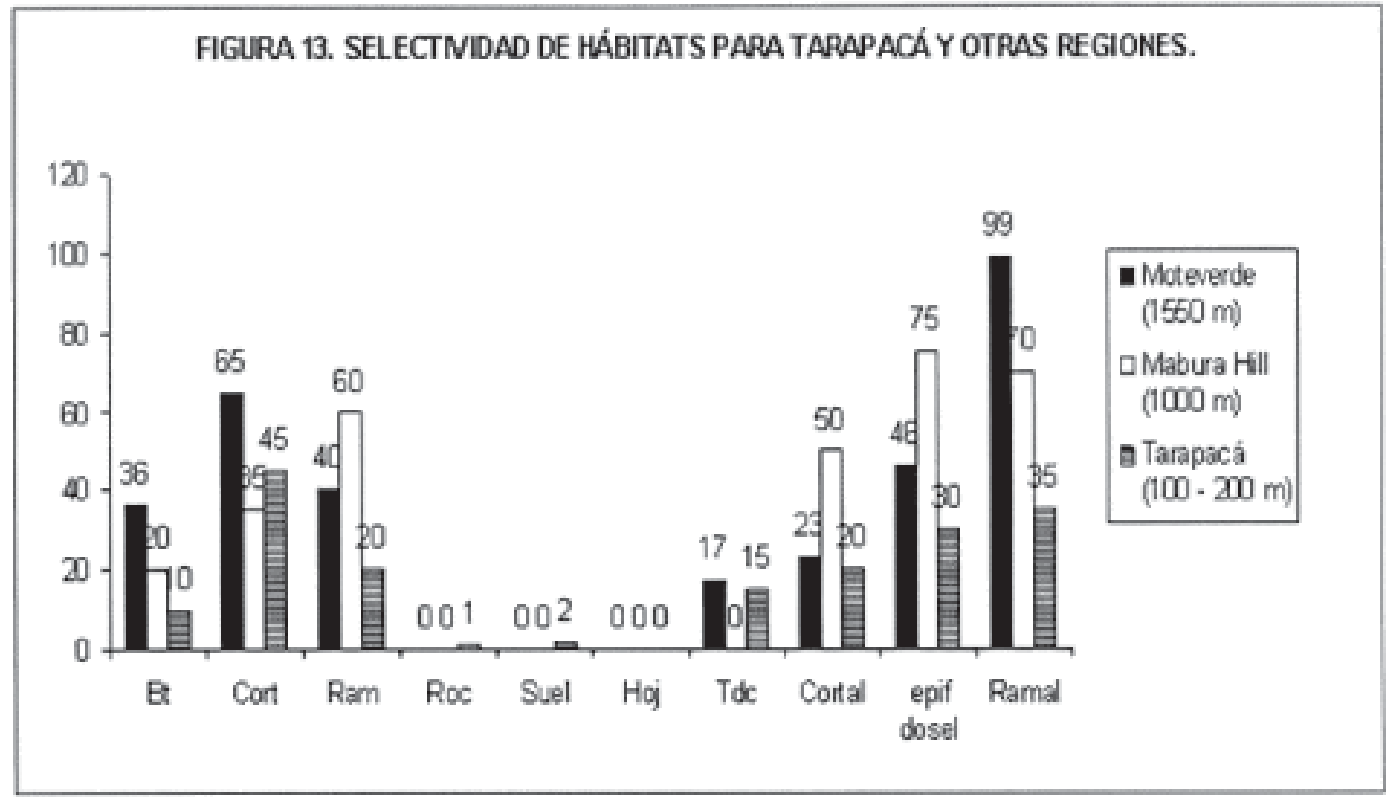

